\title{
Continuous-Time Portfolio Selection and Option Pricing under Risk-Minimization Criterion in an Incomplete Market
}

\author{
Xinfeng Ruan, ${ }^{1}$ Wenli $\mathrm{Zhu},{ }^{1}$ Jiexiang Huang, ${ }^{1}$ and Shuang $\mathrm{Li}^{2}$ \\ ${ }^{1}$ School of Economic Mathematics, Southwestern University of Finance and Economics, Chengdu 611130, China \\ ${ }^{2}$ Department of Mathematics and Statistics, Curtin University, Perth, WA 6102, Australia \\ Correspondence should be addressed to Xinfeng Ruan; ruanxinf@gmail.com
}

Received 25 June 2013; Accepted 11 September 2013

Academic Editor: Guangchen Wang

Copyright (c) 2013 Xinfeng Ruan et al. This is an open access article distributed under the Creative Commons Attribution License, which permits unrestricted use, distribution, and reproduction in any medium, provided the original work is properly cited.

\begin{abstract}
We study option pricing with risk-minimization criterion in an incomplete market where the dynamics of the risky underlying asset are governed by a jump diffusion equation. We obtain the Radon-Nikodym derivative in the minimal martingale measure and a partial integrodifferential equation (PIDE) of European call option. In a special case, we get the exact solution for European call option by Fourier transformation methods. Finally, we employ the pricing kernel to calculate the optimal portfolio selection by martingale methods.
\end{abstract}

\section{Introduction}

Option pricing problem is one of the predominant concerns in the financial market. Since the advent of the justly celebrated Black-Scholes option pricing formula in [1], there has been an increasing amount of literature describing the theory and its practice. Due to drawbacks of the Black-Scholes model which cannot explain numerous empirical facts: large, sudden movements in prices, heavy tails, and the incompleteness of markets, the concentration of losses in a few large downward moves, many option valuation models have been proposed and tested to fit those empirical facts. The jump diffusion models which have well computational and statistical features are successful to solve those drawbacks of the BlackScholes model in [2-9]. Therefore, in this paper, with those nice properties, we choose this jump diffusion model.

Different from the Black-Scholes framework, we use jump diffusion to describe the price dynamics of underlying assets and let the market of our model be incomplete; that is, it is not possible to replicate the payoff of every contingent claim by a portfolio, and there are several equivalent martingale measures. How to choose a consistent pricing measure from the set of equivalent martingale measures becomes an important problem. That means we need to find some criteria to determine one from the set of equivalent martingale measures in some economically or mathematically motivated fashion. Föllmer and Leukert (2000), Kallsen (1999), Cvitanić et al. (2001), and Bielecki and Jeanblanc (2008) in [10-13] identified a unique equivalent martingale measure by utility maximization. Then the option valuation under the minimal martingale measure was further developed by several researchers. Schweizer (1991), and Föllmer and Schweizer (1991) in [14, 15] found that in the minimal martingale measure, a unique risk-minimizing (or optimal) strategy hedging of contingent claims in incomplete market exists. In our paper, we name the criterion in the minimal martingale measure as risk-minimization criterion. Thus, in the incomplete market, option pricing is approximately possible with risk-minimization criterion. As presented in this paper, our work is based on the task of Föllmer and Schweizer in $[14,15]$, and the purpose of this paper is to find the minimal martingale measure and the measure switch of asset prices processes with jump diffusion. By using the minimal martingale measure, we obtain the Radon-Nikodym derivative which links the physical and risk-neutral densities and a PIDE of the European option. In a special case, we get the exact solution for European call option by Fourier transformation methods. Recently, Ruan et al. (2013) in [16] studied option pricing with risk-minimization criterion in an incomplete market when the dynamics of the risky 
underlying asset are governed by a jump diffusion equation with stochastic volatility.

General equilibrium framework is also a popular method to deal with the option pricing in an incomplete market. Pan (2002), Liu and Pan (2003), and Liu et al. (2005) in [17-19] derived the pricing kernel with some restrictions of jump sizes in a general equilibrium setting. Recently, Zhang et al. (2012) in [9] presented an analytical form for the pricing kernel without any distributional assumption on the jumps, the moments in physical and risk-neutral measure, respectively. In this paper, we get pricing kernel and the physical moments in and the risk-neutral physical moments without any distributional assumption on the jumps which are similar to the results of Zhang et al. (2012) [9].

Anther contribution of our paper is finding the optimal portfolio selection and terminal wealth by martingale methods. The popular methods to solve the optimal portfolio problem are stochastic control methods, which derive some complex partial differential equations (PDE). For example, see [20-23]. However, martingale methods, built around the ideas of equivalent martingale measure in complete markets, began with Harrison and Kreps (1979) in [24] and were further developed by Harrison and Pliska (1983) in [25]. They claimed that an optimal terminal wealth was first identified by solving a static optimization problem and then an efficient portfolio was obtained by replicating the optimal terminal wealth. Karatzas et al. (1991) in [26] and He and Pearson (1991) in [27] considered a Brownian model in which the number of stocks was strictly less than the dimension of the driving Brownian motion. In all these papers, it was assumed that the underlying assets satisfy just diffusion processes. In this paper, considering a jump diffusion model, we employ the pricing kernel in minimal martingale measure to degenerate a stochastic optimal terminal wealth problem into a static optimization problem.

The rest of the paper is organized as follows. In Section 2, we present the model for the underlying market and DoobMeyer decomposition of the risky asset. In Section 3, we investigate an explicit representation of the density process of the minimal martingale measure. In Section 4, we derive a PIDE with respect to the European option and present an exact solution of European call option by Fourier transformation methods in a special case. The optimal portfolio selection problem by martingale methods is studied in Section 5 and conclusions are given in Section 6.

\section{The Model}

In this paper, we consider the financial market with the following two basic assets:

(i) a bond whose price $B_{t}$ at time $t$ is given by

$$
d B_{t}=r B_{t} d t, \quad B_{0}=1
$$

(ii) a stock whose price $S_{t}$ at time $t$ is given by

$$
\frac{d S_{t}}{S_{t^{-}}}=\mu d t+\sigma d W_{t}+\int_{R_{0}}\left(e^{x}-1\right) \bar{N}(d t, d x), \quad S_{0}>0 .
$$

Here $t \in[0, T], x \in R_{0} \subset R$, constants $\mu>r>0, \sigma>0$, and $T>0$; on the filtered complete space $\left(\Omega, F,\left\{F_{t}\right\}_{t \geq 0}, P\right)$, there are $W_{t}$ which is 1-dimensional Brownian motion and $\bar{N}(d t, d x)=N(d t, d x)-v(d x) d t$ which is the compensated Poisson random measure. $N(d t, d x)$ is a Poisson random measure with compensator $v(d x)$. Additionally, $\int_{R_{0}}\left(e^{x}-1\right)^{2}$ $v(d x)<\infty$.

In this model, the jump process $\eta(t)=\int_{0}^{t} \int_{R_{0}}\left(e^{x}-1\right) \bar{N}$ $(d u, d x)$ built from a compensated Poisson random measure $\bar{N}(d t, d x)$ is the sum of its i.i.d. jumps, where the jumps occur according to a Poisson process $N(d t, d x)$. In particular, if we denote $R_{0}=(-\infty,+\infty), \bar{N}(d t, d x)=d N(t)-$ $v(d x) d t, v(d x)=\lambda f(x) d x$ where $\lambda$ is the jump intensity and $f(x)$ is distribution of the jump size $x$, then the compensated Poisson integral $\eta(t)$ can be rewritten as $\eta(t)=\sum_{i=1}^{N(t)}\left(e^{x_{i}}-\right.$ 1) $-t \int_{-\infty}^{+\infty}\left(e^{x}-1\right) f(x) d x$.

Now, we want to find a unique optimal strategy hedging of contingent claims under risk-minimization criterion. Based on [15], it is equivalent to find the minimal martingale measure from the set of equivalent martingale measures and then obtain approximate prices of contingent claims.

With the Doob-Meyer decomposition, the discounted risky asset price process, $\widehat{S}_{t}=e^{-r t} S_{t}$, is a special semimartingale and can be written as

$$
\widehat{S}_{t}=\widehat{S}_{0}+M_{t}+A_{t}
$$

with

$$
\begin{gathered}
M_{t}=\int_{0}^{t} \widehat{S}_{u^{-}} \sigma d W_{u}^{S}+\int_{0}^{t} \int_{R_{0}} \widehat{S}_{u^{-}}\left(e^{x}-1\right) \bar{N}(d u, d x), \\
A_{t}=\int_{0}^{t} \widehat{S}_{u^{-}}(\mu-r) d u,
\end{gathered}
$$

where $M_{t}$ is the martingale part of $\widehat{S}_{t}$ and $A_{t}$ is the predictable process of finite variation.

\section{Minimal Martingale Measure}

We introduce the notions of minimal martingale measure in this section. Föllmer and Schweizer (1991) [15] noticed that the optimal hedging strategy can be computed in terms of the minimal martingale measure. Furthermore, it is uniquely determined. Hence, in the minimal martingale measure, the Radon-Nikodym derivative can be found and computed. Before that, we define the minimal martingale measure.

Definition 1 (see [15]). A local martingale measure $Q$, equivalent to the original measure $P$, is called minimal if $Q=P$ on $F_{t}$ and if any square-integrable $P$-martingale $L$ which is $P$ orthogonal to $M$ remains a local martingale under $Q$.

Theorem 2 (see [15]). (i) The minimal martingale measure $Q$ is uniquely determined. 
(ii) $\widetilde{P}$ exists if and only if there exists a predictable process $\beta_{t}$ that satisfies

$$
Z_{t}=\frac{d Q}{d P}=1+\int_{0}^{t} \beta_{t} d M_{t} .
$$

Using Theorem 2, we obtain the following theorem for computing the Radon-Nikodym derivative.

Theorem 3. The Radon-Nikodym derivative in the minimal martingale measure $Q$ is

$$
\begin{aligned}
Z_{t}=\exp \{ & -\theta \sigma W_{t}-\frac{1}{2} \theta^{2} \sigma^{2} t \\
& +\int_{0}^{t} \int_{R_{0}} \ln \left(1-\theta\left(e^{x}-1\right)\right) N(d u, d x) \\
& \left.+t \int_{R_{0}} \theta\left(e^{x}-1\right) v(d x)\right\},
\end{aligned}
$$

where

$$
\theta=\frac{\mu-r}{\sigma^{2}+\int_{R_{0}}\left(e^{x}-1\right)^{2} v(d x)} .
$$

Proof. The theory of the Girsanov transformation shows that the predictable process of bounded variation can also be computed in terms of $Z_{t}$ :

$$
-d A_{t}=\frac{1}{Z_{t^{-}}} d\langle M, Z\rangle_{t} .
$$

Throughout this paper, we make use of the notations that $\langle A, B\rangle_{t}$ defined quadratic variation process between $A$ and $B$ and denote $\langle A\rangle_{t} \dot{=}\langle A, A\rangle_{t}$. Under $Q$, the predictable process of bounded variation in the Doob-Meyer decomposition of $M$ is given by

$$
\frac{1}{Z_{t^{-}}} d\langle M, Z\rangle_{t}=\frac{\beta_{t}}{Z_{t^{-}}} d\langle M\rangle_{t}=-d A_{t^{\prime}} .
$$

Using Theorem 2 and (10), we have

$$
Z_{t}=1-\int_{0}^{t} Z_{u^{-}} \frac{d A_{u}}{d\langle M\rangle_{u}} d M_{u}
$$

Denote $d Y_{u}=-\left(d A_{u} / d\langle M\rangle_{u}\right) d M_{u}$; then (11) can be written as

$$
Z_{t}=1+\int_{0}^{t} Z_{u_{-}} d Y_{u}
$$

From (4), we get

$$
\begin{aligned}
\langle M\rangle_{t} & =\left\langle\int_{0}^{t} \widehat{S}_{u^{-}} \sigma d W_{u}^{S}+\int_{0}^{t} \int_{R_{0}} \widehat{S}_{u^{-}}\left(e^{x}-1\right) \bar{N}(d u, d x)\right\rangle \\
& =\int_{0}^{t} \hat{S}_{u^{-}}^{2} \sigma^{2} d u+\int_{0}^{t} \int_{R_{0}} \widehat{S}_{u^{-}}^{2}\left(e^{x}-1\right)^{2} v(d x) d u \\
& =\int_{0}^{t} \hat{S}_{u^{-}}^{2}\left(\sigma^{2}+\int_{R_{0}}\left(e^{x}-1\right)^{2} v(d x)\right) d u .
\end{aligned}
$$

Hence

$$
\begin{aligned}
d Y_{u}= & -\frac{d A_{u}}{d\langle M\rangle_{u}} d M_{u} \\
= & -\frac{\widehat{S}_{u^{-}}(\mu-r) d u}{\widehat{S}_{u^{-}}^{2}\left(\sigma^{2}+\int_{R_{0}}\left(e^{x}-1\right)^{2} v(d x)\right) d u} \\
& \times\left[\widehat{S}_{u^{-}}\left(\sigma d W_{u}+\int_{R_{0}}\left(e^{x}-1\right) \bar{N}(d u, d x)\right)\right] \\
= & -\frac{(\mu-r)\left(\sigma d W_{u}+\int_{R_{0}}\left(e^{x}-1\right) \bar{N}(d u, d x)\right)}{\sigma^{2}+\int_{R_{0}}\left(e^{x}-1\right)^{2} v(d x)} .
\end{aligned}
$$

From (12), we know that $Z_{t}$ is the Doleans-Dade exponential. Thus, we obtain

$$
\begin{aligned}
Z_{t} & =1+\int_{0}^{t} Z_{u-} d Y_{u}, \quad Z_{0}=1, \\
d Y_{t} & =-\theta\left(\sigma d W_{t}+\int_{R_{0}}\left(e^{x}-1\right) \bar{N}(d t, d x)\right),
\end{aligned}
$$

where

$$
\theta=\frac{\mu-r}{\sigma^{2}+\int_{R_{0}}\left(e^{x}-1\right)^{2} v(d x)} .
$$

Solving (15), we obtain $Z_{t}$ in Theorem 3.

Remark 4. In the minimal martingale measure $Q$, we obtain unique Radon-Nikodym derivative which links the physical and minimal martingale measure. The Radon-Nikodym derivative is very important to get the PDE with respect to option price and to determine a pricing kernel which enables the portfolio selection problem to be solved by martingale methods. More details are presented in Section 5.

Remark 5. From the Girsanov theorem, the Brown motion in the minimal martingale measure $Q$ is

$$
\widetilde{W}_{t}=W_{t}+\theta \sigma t,
$$

and the compensatory of $N(d u, d y)$ is

$$
\begin{gathered}
\widetilde{v}(d x) d t=\left(1-\theta\left(e^{x}-1\right)\right) v(d x) d t, \\
\widetilde{N}(d t, d x)=N(d t, d x)-\widetilde{v}(d x) d t .
\end{gathered}
$$

Then (2) under the minimal martingale measure $\widetilde{P}$ is written as

$$
\begin{aligned}
\frac{d S_{t}}{S_{t^{-}}}= & \mu d t+\sigma d \widetilde{W}_{t}^{S}+\int_{R_{0}}\left(e^{x}-1\right) \widetilde{N}(d t, d x) \\
& -\theta \sigma^{2} d t-\int_{R_{0}} \theta\left(e^{x}-1\right)^{2} v(d x) d t, \quad S_{0}>0 .
\end{aligned}
$$

To guarantee that $\widehat{S}_{t}$ is a martingale under the minimal martingale measure $Q$, the following corollary is necessary. 
Corollary 6. In the minimal martingale measure $Q, \widehat{S}_{t}$ is a martingale if and only if

$$
\mu-r-\theta \sigma^{2}-\int_{R_{0}} \theta\left(e^{x}-1\right)^{2} v(d x)=0 .
$$

Proof. Substituting $S_{t}=e^{-r t} \widehat{S}_{t}$ into (19), since $\widehat{S}_{t}$ is a martingale, the drift term must be identical to zero. Then we can get (20).

Remark 7. Using (20), (19) in the minimal martingale measure $Q$ is written as

$$
\frac{d S_{t}}{S_{t^{-}}}=r_{t} d t+\sigma d \widetilde{W}_{t}^{S}+\int_{R_{0}}\left(e^{x}-1\right) \widetilde{N}(d t, d x), \quad S_{0}>0 .
$$

The minimal martingale measure $Q$ is a risk-neutral measure.

\section{The Pricing Formula for European Call Option}

In the minimal martingale measure $Q$, the price of the European call option $C\left(t, S_{t}\right)$ at time $t$ with strike price $K$ and maturity date $T$ is given by

$$
C\left(t, S_{t}\right)=E^{Q}\left[e^{-r(T-t)}\left(S_{T}-K\right)^{+} \mid F_{t}\right],
$$

and $C\left(T, S_{T}\right)=\left(S_{T}-K\right)^{+}$.

By the fact that the discounted price of the European call option is a martingale under $Q$, we can obtain the following theorem.

Theorem 8. In the minimal martingale measure $Q$, the price of the European call option satisfies the following PIDE:

$$
\begin{aligned}
0= & -r C\left(t, S_{t}\right)+\frac{\partial C}{\partial t}+\frac{\partial C}{\partial S} r S_{t^{-}}+\frac{1}{2} \frac{\partial^{2} C}{\partial S^{2}} \sigma^{2} S_{t^{-}}^{2} \\
& +\int_{R_{0}}\left(C\left(t, S_{t^{-}} e^{x}\right)-C\left(t, S_{t^{-}}\right)-\left(e^{x}-1\right) \frac{\partial C}{\partial S} S_{t^{-}}\right) \widetilde{v}(d x)
\end{aligned}
$$

and $C\left(T, S_{T}\right)=\left(S_{T}-K\right)^{+}$.

Proof. The total derivative of the discounted option price is

$$
\begin{aligned}
& d\left(e^{-r t} C\left(t, S_{t}\right)\right) \\
& =-r_{t} e^{-r t} C\left(t, S_{t}\right) d t+e^{-r t} \frac{\partial C}{\partial t} d t \\
& +e^{-r t} \frac{\partial C}{\partial S} d S^{C}+\frac{1}{2} e^{-r t} \frac{\partial^{2} C}{\partial S^{2}} d S^{C} d S^{C} \\
& +e^{-r t} \int_{R_{0}}\left(C\left(t, S_{t^{-}} y\right)-C\left(t, S_{t^{-}}\right)\right) N(d t, d x) \\
& =e^{-r t}\left\{-r_{t} C\left(t, S_{t}\right) d t+\frac{\partial C}{\partial t} d t+\frac{\partial C}{\partial S} S_{t^{-}}\right. \\
& \times\left(r d t+\sigma d \widetilde{W}_{t}-\int_{R_{0}}\left(e^{x}-1\right) \widetilde{v}(d x) d t\right)
\end{aligned}
$$

$$
\begin{aligned}
& +\frac{1}{2} \frac{\partial^{2} C}{\partial S^{2}} \sigma^{2} S_{t^{-}}^{2} d t \\
& +\int_{R_{0}}\left(C\left(t, S_{t^{-}} e^{x}\right)-C\left(t, S_{t^{-}}\right)\right) \widetilde{N}(d t, d x) \\
& \left.+\int_{R_{0}}\left(C\left(t, S_{t^{-}} e^{x}\right)-C\left(t, S_{t^{-}}\right)\right) \widetilde{v}(d x) d t\right\} \\
& =e^{-r t}\left\{-r C\left(t, S_{t}\right)+\frac{\partial C}{\partial t}+\frac{\partial C}{\partial S} r_{t} S_{t^{-}}+\frac{1}{2} \frac{\partial^{2} C}{\partial S^{2}} \sigma_{t}^{2} S_{t^{-}}^{2}\right. \\
& +\int_{R_{0}}\left(C\left(t, S_{t^{-}} e^{x}\right)-C\left(t, S_{t^{-}}\right)\right. \\
& \left.\left.-\left(e^{x}-1\right) \frac{\partial C}{\partial S} S_{t^{-}}\right) \widetilde{v}(d x)\right\} d t \\
& +e^{-r t}\left\{\frac{\partial C}{\partial S} S_{t^{-}} \sigma d \widetilde{W}_{t}^{S}\right. \\
& \left.+\int_{R_{0}}\left(C\left(t, S_{t^{-}} e^{x}\right)-C\left(t, S_{t^{-}}\right)\right) \tilde{N}(d t, d x)\right\} .
\end{aligned}
$$

We make the drift term be zero, since the discounted price of the European put option is a martingale. Then we obtain the equation in Theorem 8.

It is difficult to get the solution of European call option $C\left(t, S_{t}\right)$ from (23). Therefore, we degenerate the model (2) into a special case with following assumption to get an exact solution of the option price.

Assumption 9. (i) In physical measure $P$, we denote $R_{0}=$ $(-\infty,+\infty), \bar{N}(d t, d x)=d N(t)-\lambda f(x) d x$, where $\lambda$ is the jump intensity and $f(x)$ is distribution of the jump size $x$. Moreover, we denote $E[\cdot]$ as expectation operator in physical measure $P$.

(ii) In the minimal martingale measure $Q$, using Remark 4, we have $\widetilde{N}(d t, d x)=d N(t)-\lambda \widetilde{f}(x) d x$, where $\tilde{f}(x)=\left(1-\theta\left(e^{x}-1\right)\right) f(x)$ and denote $E^{\mathrm{Q}}[\cdot]$ as expectation operator in this measure.

Then the stock price process (1) in physical measure can be written as

$$
\begin{gathered}
\frac{d S_{t}}{S_{t^{-}}}=\mu d t-\lambda E\left[\left(e^{x}-1\right)\right] d t+\sigma d W_{t} \\
+\left(e^{x}-1\right) d N(t), \quad S_{0}>0,
\end{gathered}
$$

and the Radon-Nikodym derivative can be written as

$$
\begin{aligned}
Z_{t}=\exp \{ & -\theta \sigma W_{t}+\theta \lambda E\left[\left(e^{x}-1\right)\right] t \\
& \left.-\frac{1}{2} \theta^{2} \sigma^{2} t+\sum_{i=1}^{N(t)} \ln \left(1-\theta\left(e^{x_{i}}-1\right)\right)\right\},
\end{aligned}
$$

where $\theta=(\mu-r) /\left(\sigma^{2}+\lambda E\left[\left(e^{x}-1\right)^{2}\right]\right)$. 
The stock price process (21) in the minimal martingale measure $Q$ can be written as

$$
\begin{gathered}
\frac{d S_{t}}{S_{t^{-}}}=r_{t} d t-\lambda E^{Q}\left[\left(e^{x}-1\right)\right] d t+\sigma d \widetilde{W}_{t} \\
+\left(e^{x}-1\right) d N(t), \quad S_{0}>0,
\end{gathered}
$$

and the price of the European call option satisfies the following equation:

$$
\begin{aligned}
0= & -r C(t, S)+\frac{\partial C}{\partial t}+\left(r-\lambda E^{Q}\left[\left(e^{x}-1\right)\right]\right) \frac{\partial C}{\partial S} S \\
& +\frac{1}{2} \frac{\partial^{2} C}{\partial S^{2}} \sigma^{2} S^{2}+\lambda\left\{E^{Q}\left[\left(C\left(t, S e^{x}\right)\right]-C(t, S)\right\}\right.
\end{aligned}
$$

and $C\left(T, S_{T}\right)=\left(S_{T}-K\right)^{+}$.

Using Fourier transformation methods, it is easy to obtain the solution of (28). The solution is shown in the following theorem.

Theorem 10. The pricing formula of European call option under Assumption 9 is given by

$$
C(S, \tau)=-\frac{1}{2 \pi} \int_{i k_{i}-\infty}^{i k_{i}+\infty} e^{-i k \ln S} \frac{K^{i k+1}}{k^{2}-i k} e^{A \tau} d k,
$$

where

$$
\begin{aligned}
A= & -(1+i k) r+i k \lambda E^{Q}\left(e^{x}-1\right)-\frac{1}{2} \sigma^{2}\left(k^{2}-i k\right) \\
& +\lambda E^{Q}\left[e^{-i k x}-1\right], \quad \tau=T-t .
\end{aligned}
$$

Proof. First, we denote $X=\ln S$ and $g(X, t):=c\left(e^{X}, t\right)=$ $C(S, t)$; then, the PDE (28) can be rewritten as

$$
\begin{aligned}
-g_{t}= & -r g+\frac{1}{2} \sigma^{2} g_{X X}+\left[r-\lambda E^{Q}\left(e^{x}-1\right)-\frac{1}{2} \sigma^{2}\right] g_{X} \\
& +\lambda\left\{E^{Q}[g(X+x, t)]-g(X, t)\right\}, \\
g(X, T)= & \left(e^{X}-K\right)^{+} .
\end{aligned}
$$

Let $G(k, V, t)$ be Fourier transform of $g(X, V, t)$,

$$
G(k, t)=\int_{-\infty}^{+\infty} e^{i k X} g(X, t) d X
$$

and $G(k, T)=-K^{i k+1} /\left(k^{2}-i k\right)$.

Denote $k=k_{r}+i k_{i}, \tau=T-t$; the inverse Fourier transform is given by

$$
g(X, t)=\frac{1}{2 \pi} \int_{i k_{i}-\infty}^{i k_{i}+\infty} e^{-i k X} G(k, t) d k .
$$

Then, the PDE (31) can be rewritten as

$$
\begin{gathered}
G_{\tau}=-(1+i k) r G+i k \lambda E^{\mathrm{Q}}\left(e^{x}-1\right) G \\
-\frac{1}{2} \sigma^{2}\left(k^{2}-i k\right) G+\lambda E^{\mathrm{Q}}\left[e^{-i k x}-1\right] G \\
G(k, 0)=-\frac{K^{i k+1}}{k^{2}-i k} .
\end{gathered}
$$

The solution of (34) is given by

$$
G(k, \tau)=-\frac{K^{i k+1}}{k^{2}-i k} e^{A \tau},
$$

where $A=-(1+i k) r+i k \lambda E^{Q}\left(e^{x}-1\right)-(1 / 2) \sigma^{2}\left(k^{2}-i k\right)+$ $\lambda E^{Q}\left[e^{-i k x}-1\right]$.

Hence,

$$
g(X, \tau)=-\frac{1}{2 \pi} \int_{i k_{i}-\infty}^{i k_{i}+\infty} e^{-i k X} \frac{K^{i k+1}}{k^{2}-i k} e^{A \tau} d k .
$$

Then

$$
C(S, \tau)=-\frac{1}{2 \pi} \int_{i k_{i}-\infty}^{i k_{i}+\infty} e^{-i k \ln S} \frac{K^{i k+1}}{k^{2}-i k} e^{A \tau} d k
$$

Note 1. Although the pricing formula (29) contains a complex integral, the result is real.

Then, we try to find the relationship of central moments between the physical measure and risk-neutral measure which can help us to study the negative variance risk premium, the implied volatility smirk, and the prediction of realized skewness. The typical literature is Bakshi et al. (2003) in [28], Carr and Wu (2009) in [29], and Neuberger (2012) in [30].

However, to the best of our knowledge, except Zhang et al. (2012) in [9], there is no literature that studies this relationship. First we present a lemma from Zhang et al. (2012) in [9] to prove Proposition 12 which presents the relationship of central moments between the physical measure and riskneutral measure $Q$.

Lemma 11. The first moment, and the second, third, and fourth central moments of the continuously compounded return within $\tau, R_{\tau}=\ln S_{T} / S_{t}$, in the risk-neutral measure $Q$ are given by

$$
\begin{gathered}
E_{t}^{\mathrm{Q}}\left(R_{\tau}\right)=\left[\mu-\frac{1}{2} \sigma^{2}-\lambda^{\mathrm{Q}} E^{\mathrm{Q}}\left(e^{x}-1-x\right)\right] \tau \\
E_{t}^{\mathrm{Q}}\left[R_{\tau}-E_{t}^{\mathrm{Q}}\left(R_{\tau}\right)\right]^{2}=\tau \sigma^{2}+\lambda^{\mathrm{Q}} \tau\left[\left(\mu_{x}^{\mathrm{Q}}\right)^{2}+\operatorname{Var}^{\mathrm{Q}}(x)\right] \\
E_{t}^{\mathrm{Q}}\left[R_{\tau}-E_{t}^{\mathrm{Q}}\left(R_{\tau}\right)\right]^{3} \\
=\lambda^{\mathrm{Q}} \tau\left[\left(\mu_{x}^{\mathrm{Q}}\right)^{3}+3 \mu_{x}^{\mathrm{Q}} \operatorname{Var}^{\mathrm{Q}}(x)+3 r d^{\mathrm{Q}}(x)\right] \\
E_{t}^{\mathrm{Q}}\left[R_{\tau}-E_{t}^{\mathrm{Q}}\left(R_{\tau}\right)\right]^{4} \\
=3\left(\lambda^{\mathrm{Q}} \tau\left[\left(\mu_{x}^{\mathrm{Q}}\right)^{2}+\operatorname{Var}^{\mathrm{Q}}(x)\right]+\tau \sigma^{2}\right)^{2} \\
+\lambda^{\mathrm{Q}}\left[\left(\mu_{x}^{\mathrm{Q}}\right)^{4}+6\left(\mu_{x}^{\mathrm{Q}}\right)^{2} \operatorname{Var}^{\mathrm{Q}}(x)\right. \\
\left.+4 \mu_{x}^{\mathrm{Q}} 3 r d^{\mathrm{Q}}(x)+4 t h^{\mathrm{Q}}(x)\right]
\end{gathered}
$$


where $\mu_{x}^{\mathrm{Q}}=E^{\mathrm{Q}}(x)$ is the first moment and $\operatorname{Var}^{\mathrm{Q}}(x)=E^{\mathrm{Q}}[(x-$ $\left.\left.\mu_{x}^{\mathrm{Q}}\right)^{2}\right], 3 r d^{\mathrm{Q}}(x)=E^{\mathrm{Q}}\left[\left(x-\mu_{x}^{\mathrm{Q}}\right)^{3}\right]$, and $4 t h^{\mathrm{Q}}(x)=E^{\mathrm{Q}}\left[\left(x-\mu_{x}^{\mathrm{Q}}\right)^{4}\right]$ are the second, third, and fourth central moments in the riskneutral measure of random number $x . \lambda^{\mathrm{Q}}$ is the jump intensity in the risk-neutral measure.

The first moment and the second, third, and fourth central moments of the continuously compounded return in the physical measure are given by

$$
\begin{gathered}
E_{t}\left(R_{\tau}\right)=\left[\mu-\frac{1}{2} \sigma^{2}-\lambda E\left(e^{x}-1-x\right)\right] \tau, \\
E_{t}\left[R_{\tau}-E_{t}\left(R_{\tau}\right)\right]^{2}=\tau \sigma^{2}+\lambda \tau\left[\mu_{x}^{2}+\operatorname{Var}(x)\right], \\
E_{t}\left[R_{\tau}-E_{t}\left(R_{\tau}\right)\right]^{3}=\lambda \tau\left[\mu_{x}^{3}+3 \mu_{x} \operatorname{Var}(x)+3 r d(x)\right], \\
E_{t}\left[R_{\tau}-E_{t}\left(R_{\tau}\right)\right]^{4} \\
=3\left(\lambda \tau\left[\mu_{x}^{2}+\operatorname{Var}(x)\right]+\tau \sigma^{2}\right)^{2} \\
+\lambda \tau\left[\mu_{x}^{4}+6 \mu_{x}^{2} \operatorname{Var}(x)+4 \mu_{x} 3 r d(x)+4 t h(x)\right],
\end{gathered}
$$

where $\mu_{x}=E(x)$ is the first moment and $\operatorname{Var}(x)=E[(x-$ $\left.\left.\mu_{x}\right)^{2}\right], 3 r d(x)=E\left[\left(x-\mu_{x}\right)^{3}\right]$, and $4 \operatorname{th}(x)=E\left[\left(x-\mu_{x}\right)^{4}\right]$ are second, third, and fourth central moments in the physical measure of random number $x . \lambda$ is the jump intensity in the physical measure.

Proof. See Zhang et al. (2012) in [9].

Proposition 12. The first moment and the second, third, and fourth central moments of the continuously compounded return within $\tau, R_{\tau}=\ln S_{T} / S_{t}$, in the physical measure are given by

$$
\begin{gathered}
E_{t}\left(R_{\tau}\right)=\mu-\frac{1}{2} \sigma^{2}-\lambda E\left[\left(e^{x}-1-x\right)\right], \\
E_{t}\left[R_{\tau}-E_{t}\left(R_{\tau}\right)\right]^{2}=\sigma^{2} \tau+\lambda \tau E\left[x^{2}\right], \\
E_{t}\left[R_{\tau}-E_{t}\left(R_{\tau}\right)\right]^{3}=\lambda \tau E\left[x^{3}\right], \\
E_{t}\left[R_{\tau}-E_{t}\left(R_{\tau}\right)\right]^{4}=\lambda \tau E\left[x^{4}\right]+3\left(\lambda \tau E\left[x^{2}\right]+\tau \sigma^{2}\right)^{2} .
\end{gathered}
$$

The first moment and the second, third, and fourth central moments of the continuously compounded return in the minimal martingale measure $Q$ are given by

$$
\begin{gathered}
E_{t}^{\mathrm{Q}}\left(R_{\tau}\right)=\mu-\frac{1}{2} \sigma^{2}-\lambda E\left[\left(1-\theta\left(e^{x}-1\right)\right)\left(e^{x}-1-x\right)\right], \\
E_{t}^{\mathrm{Q}}\left[R_{\tau}-E_{t}^{\mathrm{Q}}\left(R_{\tau}\right)\right]^{2}=\sigma^{2} \tau+\lambda \tau E\left[\left(1-\theta\left(e^{x}-1\right)\right) x^{2}\right], \\
E_{t}^{\mathrm{Q}}\left[R_{\tau}-E_{t}^{\mathrm{Q}}\left(R_{\tau}\right)\right]^{3}=\lambda \tau E\left[\left(1-\theta\left(e^{x}-1\right)\right) x^{3}\right], \\
E_{t}^{\mathrm{Q}}\left[R_{\tau}-E_{t}^{\mathrm{Q}}\left(R_{\tau}\right)\right]^{4} \\
=\lambda \tau E\left[\left(1-\theta\left(e^{x}-1\right)\right) x^{4}\right] \\
+3\left(\tau \sigma^{2}+\lambda \tau E\left[\left(1-\theta\left(e^{x}-1\right)\right) x^{2}\right]\right)^{2} .
\end{gathered}
$$

Proof. To compute central moments in the physical measure, we have

$$
\begin{gathered}
\mu_{x}=E(x), \\
\operatorname{Var}(x)=E\left[\left(x-\mu_{x}\right)^{2}\right]=E\left[x^{2}\right]-\mu_{x}^{2}, \\
3 r d(x)=E\left[\left(x-\mu_{x}\right)^{3}\right] \\
=E\left[x^{3}-3 x^{2} \mu_{x}+3 x \mu_{x}^{2}-\mu_{x}^{3}\right] \\
=E\left[x^{3}\right]-3 \mu_{x}^{2} E\left[x^{2}\right]+2 \mu_{x}^{3}, \\
4 \operatorname{th}(x)=E\left[\left(x-\mu_{x}\right)^{4}\right] \\
=E\left[x^{4}-4 x^{3} \mu_{x}+6 x^{2} \mu_{x}^{2}-4 x \mu_{x}^{3}+\mu_{x}^{4}\right] \\
=E\left[x^{4}\right]-4 \mu_{x} E\left[x^{3}\right]+6 \mu_{x}^{2} E\left[x^{2}\right]-3 \mu_{x}^{4} .
\end{gathered}
$$

Then, from Lemma 11, we have

$$
\begin{gathered}
E_{t}\left(R_{\tau}\right)=\mu-\frac{1}{2} \sigma^{2}-\lambda E\left[\left(e^{x}-1-x\right)\right], \\
E_{t}\left[R_{\tau}-E_{t}\left(R_{\tau}\right)\right]^{2}=\sigma^{2} \tau+\lambda \tau\left[\mu_{x}^{2}+\operatorname{Var}(x)\right] \\
=\sigma^{2} \tau+\lambda \tau E\left[x^{2}\right], \\
E_{t}\left[R_{\tau}-E_{t}\left(R_{\tau}\right)\right]^{3}=\lambda \tau\left[\mu_{x}^{3}+3 \mu_{x} \operatorname{Var}(x)+3 r d(x)\right] \\
=\lambda \tau E\left[x^{3}\right], \\
E_{t}\left[R_{\tau}-E_{t}\left(R_{\tau}\right)\right]^{4}=3\left(\lambda \tau\left[\mu_{x}^{2}+\operatorname{Var}(x)\right]+\tau \sigma^{2}\right)^{2} \\
+\lambda \tau\left[\mu_{x}^{4}+6 \mu_{x}^{2} \operatorname{Var}(x)\right. \\
\left.+4 \mu_{x} 3 r d(x)+4 t h(x)\right] \\
=\lambda \tau E\left[x^{4}\right]+3\left(\lambda \tau E\left[x^{2}\right]+\tau \sigma^{2}\right)^{2} .
\end{gathered}
$$

To compute central moments in the physical measure, we get

$$
\begin{aligned}
\mu_{x}^{\mathrm{Q}} & =E^{\mathrm{Q}}(x)=E\left[\left(1-\theta\left(e^{x}-1\right)\right) x\right], \\
\operatorname{Var}^{\mathrm{Q}}(x) & =E^{\mathrm{Q}}\left[\left(x-\mu_{x}^{\mathrm{Q}}\right)^{2}\right] \\
& =E\left[\left(1-\theta\left(e^{x}-1\right)\right)\left(x-\mu_{x}^{\mathrm{Q}}\right)^{2}\right] \\
& =\frac{E\left[\left(1-\theta\left(e^{x}-1\right)\right)\left(x^{2}-2 \mu_{x}^{\mathrm{Q}} x+\left(\mu_{x}^{\mathrm{Q}}\right)^{2}\right)\right]}{E\left(e^{-\gamma x}\right)} \\
& =E\left[\left(1-\theta\left(e^{x}-1\right)\right) x^{2}\right]-\left(\mu_{x}^{\mathrm{Q}}\right)^{2},
\end{aligned}
$$




$$
\begin{aligned}
3 r d^{\mathrm{Q}}(x)= & E^{\mathrm{Q}}\left[\left(x-\mu_{x}^{\mathrm{Q}}\right)^{3}\right] \\
= & E\left[\left(1-\theta\left(e^{x}-1\right)\right)\left(x-\mu_{x}^{\mathrm{Q}}\right)^{3}\right] \\
= & E\left[\left(1-\theta\left(e^{x}-1\right)\right)\right. \\
& \left.\times\left(x^{3}-3 x^{2} \mu_{x}^{\mathrm{Q}}+3 x\left(\mu_{x}^{\mathrm{Q}}\right)^{2}-\left(\mu_{x}^{\mathrm{Q}}\right)^{3}\right)\right] \\
= & E\left[\left(1-\theta\left(e^{x}-1\right)\right) x^{3}\right]-3 \mu_{x}^{\mathrm{Q}} E \\
& \times\left[\left(1-\theta\left(e^{x}-1\right)\right) x^{2}\right]+2\left(\mu_{x}^{\mathrm{Q}}\right)^{3}, \\
4 t h^{\mathrm{Q}}(x)= & E^{\mathrm{Q}}\left[\left(x-\mu_{x}^{\mathrm{Q}}\right)^{4}\right] \\
= & E\left[\left(1-\theta\left(e^{x}-1\right)\right)\left(x-\mu_{x}^{\mathrm{Q}}\right)^{4}\right] \\
= & E\left[( 1 - \theta ( e ^ { x } - 1 ) ) \left(x^{4}-4 x^{3} \mu_{x}^{\mathrm{Q}}+6 x^{2}\left(\mu_{x}^{\mathrm{Q}}\right)^{2}\right.\right. \\
& \left.\left.\quad-4 x\left(\mu_{x}^{\mathrm{Q}}\right)^{3}+\left(\mu_{x}^{\mathrm{Q}}\right)^{4}\right)\right] \\
= & E\left[\left(1-\theta\left(e^{x}-1\right)\right) x^{4}\right] \\
& -4 E\left[\left(1-\theta\left(e^{x}-1\right)\right) x^{3}\right] \mu_{x}^{\mathrm{Q}} \\
& +6 E\left[\left(1-\theta\left(e^{x}-1\right)\right) x^{2}\right]\left(\mu_{x}^{\mathrm{Q}}\right)^{2}-3\left(\mu_{x}^{\mathrm{Q}}\right)^{4} .
\end{aligned}
$$

Then, from (25), we have

$$
\begin{aligned}
E_{t}^{\mathrm{Q}}\left(R_{\tau}\right)= & \mu-\frac{1}{2} \sigma^{2}-\lambda E \\
& \times\left[\left(1-\theta\left(e^{x}-1\right)\right)\left(e^{x}-1-x\right)\right], \\
E_{t}^{\mathrm{Q}}\left[R_{\tau}-\right. & \left.E_{t}^{\mathrm{Q}}\left(R_{\tau}\right)\right]^{2} \\
= & \sigma^{2} \tau+\lambda^{\mathrm{Q}} \tau\left[\left(\mu_{x}^{\mathrm{Q}}\right)^{2}+\operatorname{Var}^{\mathrm{Q}}(x)\right] \\
= & \sigma^{2} \tau+\lambda \tau E\left[\left(1-\theta\left(e^{x}-1\right)\right) x^{2}\right], \\
E_{t}^{\mathrm{Q}}\left[R_{\tau}-\right. & \left.E_{t}^{\mathrm{Q}}\left(R_{\tau}\right)\right]^{3} \\
= & \lambda^{\mathrm{Q}} \tau\left[\left(\mu_{x}^{\mathrm{Q}}\right)^{3}+3 \mu_{x}^{\mathrm{Q}} \operatorname{Var}^{\mathrm{Q}}(x)+3 r d^{\mathrm{Q}}(x)\right] \\
= & \lambda \tau\left\{\left(\mu_{x}^{\mathrm{Q}}\right)^{3}+3 \mu_{x}^{\mathrm{Q}}\right. \\
& \times\left[E\left[\left(1-\theta\left(e^{x}-1\right)\right) x^{2}\right]-\left(\mu_{x}^{\mathrm{Q}}\right)^{2}\right] \\
+ & {\left[E\left[\left(1-\theta\left(e^{x}-1\right)\right) x^{3}\right]-3 \mu_{x}^{\mathrm{Q}} E\right.} \\
& \left.\left.\times\left[\left(1-\theta\left(e^{x}-1\right)\right) x^{2}\right]+2\left(\mu_{x}^{\mathrm{Q}}\right)^{3}\right]\right\} \\
= & \lambda \tau E\left[\left(1-\theta\left(e^{x}-1\right)\right) x^{3}\right],
\end{aligned}
$$

$$
\begin{aligned}
E_{t}^{\mathrm{Q}}\left[R_{\tau}-\right. & \left.E_{t}^{\mathrm{Q}}\left(R_{\tau}\right)\right]^{4} \\
= & 3\left(\lambda \tau\left[\left(\mu_{x}^{\mathrm{Q}}\right)^{2}+\operatorname{Var}^{\mathrm{Q}}(x)\right]+\sigma^{2} \tau\right)^{2} \\
& +\lambda^{\mathrm{Q}} \tau\left[\left(\mu_{x}^{\mathrm{Q}}\right)^{4}+6\left(\mu_{x}^{\mathrm{Q}}\right)^{2} \operatorname{Var}^{\mathrm{Q}}(x)\right. \\
& \left.+4 \mu_{x}^{\mathrm{Q}} 3 r d^{\mathrm{Q}}(x)+4 t h^{\mathrm{Q}}(x)\right] \\
= & \lambda \tau E\left[\left(1-\theta\left(e^{x}-1\right)\right) x^{4}\right] \\
& +3\left(\lambda \tau E\left[\left(1-\theta\left(e^{x}-1\right)\right) x^{2}\right]+\sigma^{2} \tau\right)^{2} .
\end{aligned}
$$

\section{Portfolio Selection}

Under Assumption 9, we study the portfolio selection problem by martingale methods in this section. We first suppose that the portfolio $(\pi(t), 1-\pi(t))$ which is the proportion of money invested in the stock market and bond market, respectively, the wealth process $y(t)=y^{\omega}(t)$, and the initial wealth $y(0)=y_{0}>0$ satisfy the equation as follows:

$$
\begin{gathered}
\frac{d y(t)}{y(t)}=r d t+\pi(t)\left[(\mu-r) d t-\lambda E\left[\left(e^{x}-1\right)\right] d t\right. \\
\left.+\sigma d W_{t}+\left(e^{x}-1\right) d N(t)\right] \\
y(0)=y_{0} .
\end{gathered}
$$

The investor maximizes his/her expected utility,

$$
\max _{\pi(t)} E[U(y(T))] .
$$

We suppose $U(y(T))=\gamma^{-1} y(T)^{\gamma}, \gamma<1, \gamma \neq 0$; then the problem which is to find portfolio to maximize the expected utility is given by the following.

\section{Problem 13. Consider}

$$
\max E\left[\gamma^{-1} y(T)^{\gamma}\right]
$$

subject to $\quad(y(\cdot), \pi(\cdot))$ satisfies $(46)$,

$\pi(\cdot)$ is admissible and tame.

We define the pricing kernel as follows:

$$
\begin{aligned}
& H(t)= e^{-r t} Z_{t} \\
&=\exp \left\{-\theta \sigma W_{t}-r t-\frac{1}{2} \theta^{2} \sigma^{2} t\right. \\
&\left.+\theta \lambda E\left[\left(e^{x}-1\right)\right] t+\sum_{i=1}^{N(t)} \ln \left(1-\theta\left(e^{x_{i}}-1\right)\right)\right\} .
\end{aligned}
$$


Then

$$
\begin{gathered}
d H(t)=-H(t)\left[\theta \sigma d W_{t}+r d t-\theta \lambda E\left[\left(e^{x}-1\right)\right] d t\right. \\
\left.+\theta\left(e^{x}-1\right) d N(t)\right] .
\end{gathered}
$$

Using $\mu-r=\theta \sigma^{2}+\theta \lambda E\left[\left(e^{x}-1\right)^{2}\right]$ and (46), we have

$$
\begin{aligned}
d(y(t) & H(t)) \\
= & y(t) d H(t)+H(t) d y(t)+d y(t) d H(t) \\
= & -y(t) H(t)\left(\theta \sigma d W_{t}+r d t-\theta \lambda E\right. \\
& \times\left[\left(e^{x}-1\right)\right] d t \\
& \left.+\theta\left(e^{x}-1\right) d N(t)\right) \\
+ & r H(t) y(t) d t+H(t) \pi(t) y(t) \\
\times & \left((\mu-r) d t-\lambda E\left[\left(e^{x}-1\right)\right] d t+\sigma d W_{t}\right. \\
& \left.+\left(e^{x}-1\right) d N(t)\right) \\
- & H(t) y(t) \theta \sigma^{2} d t-\pi(t) y(t) \\
& \times H(t) \theta \lambda E\left[\left(e^{x}-1\right)^{2}\right] \\
= & y(t) H(t)(\sigma \pi(t)-\sigma \theta) d W_{t}-y(t) H(t) \\
\times & \left(-\theta \lambda E\left[\left(e^{x}-1\right)\right] d t+\theta\left(e^{x}-1\right) d N(t)\right) \\
+ & H(t) \pi(t) y(t) \\
\times & \left(-\lambda E\left[\left(e^{x}-1\right)\right] d t+\left(e^{x}-1\right) d N(t)\right) .
\end{aligned}
$$

It suggests that $y(t) H(t)$ is a martingale in physical measure.

In order to solve Problem 13 one needs only first to solve the following optimization problem in the terminal wealth. $Y$ is predictable stochastic process.

Problem 14. Consider

$$
\begin{aligned}
& \max E\left[\gamma^{-1} Y^{\gamma}\right] \\
& \text { subject to } E[H(T) Y]=y_{0} .
\end{aligned}
$$

Solving Problem 14, we obtain the following theorem.

Theorem 15. If $Y^{*}$ is optimal for Problem 14, then $Y^{*}$ is also optimal for Problem 13, where

$$
\begin{aligned}
& Y^{*}=y_{0} \exp \{-\frac{1}{\gamma-1} \theta \sigma W_{T}+r T-\frac{(2 \gamma-1) \theta^{2} \sigma^{2}}{2(\gamma-1)^{2}} T \\
&-\theta \lambda E\left[\left(e^{x}-1\right)\right] T \\
&\left.-\lambda E\left[\left(1-\theta\left(e^{x}-1\right)\right)^{\gamma /(\gamma-1)}-1\right] T\right\} \\
& \cdot \exp \left\{\sum_{i=1}^{N(T)} \frac{1}{\gamma-1} \ln \left(1-\theta\left(e^{x_{i}}-1\right)\right)\right\}
\end{aligned}
$$$$
\text { and } \pi^{*}(t)=(1 /(1-\gamma))\left((\mu-r) /\left(\sigma^{2}+\lambda E\left[\left(e^{x}-1\right)^{2}\right]\right)\right) \text {. }
$$

Proof. The Lagrangian function of Problem 14 is given by

$$
L(Y, \lambda)=E\left[\gamma^{-1} Y^{\gamma}+\lambda\left(y_{0}-H(T) Y\right)\right]
$$

where $\lambda$ is the Lagrangian multiplier. Equating the derivatives of the $L(Y, \lambda)$ with respect to $Y$ and $\lambda$, respectively, to zero, we have

$$
\begin{gathered}
\frac{\partial L(Y, \lambda)}{\partial Y}=E\left[Y^{\gamma-1}-\lambda H(T)\right]=0 \\
L(Y, \lambda)=y_{0}-E[H(T) Y]=0 .
\end{gathered}
$$

From (55), we get

$$
Y^{*}=\lambda^{1 /(\gamma-1)} H(T)^{1 /(\gamma-1)} .
$$

Substituting (57) into (56), we obtain

$$
\lambda^{1 /(\gamma-1)}=\frac{y_{0}}{E\left[H(T)^{\gamma /(\gamma-1)}\right]},
$$

since

$$
\begin{aligned}
& E\left[H(T)^{\gamma /(\gamma-1)}\right] \\
& =E \exp \left\{-\frac{\gamma}{\gamma-1} \theta \sigma W_{T}-\frac{\gamma}{\gamma-1} r T\right. \\
& -\frac{1}{2} \frac{\gamma}{\gamma-1} \theta^{2} \sigma^{2} T+\frac{\gamma}{\gamma-1} \theta \lambda E\left[\left(e^{x}-1\right)\right] T \\
& \left.+\sum_{i=1}^{N(T)} \frac{\gamma}{\gamma-1} \ln \left(1-\theta\left(e^{x_{i}}-1\right)\right)\right\} \\
& =E \exp \left\{\frac{\gamma \theta^{2} \sigma^{2}}{2(\gamma-1)^{2}} T-\frac{\gamma r T}{\gamma-1}+\frac{\gamma}{\gamma-1} \theta \lambda E\left[\left(e^{x}-1\right)\right] T\right\} \\
& \cdot E \exp \left\{\sum_{i=1}^{N(T)} \frac{\gamma}{\gamma-1} \ln \left(1-\theta\left(e^{x_{i}}-1\right)\right)\right\} \\
& =\exp \left\{\frac{\gamma \theta^{2} \sigma^{2}}{2(\gamma-1)^{2}} T-\frac{\gamma r T}{\gamma-1}+\frac{\gamma}{\gamma-1} \theta \lambda E\left[\left(e^{x}-1\right)\right] T\right\} \\
& \cdot \exp \left\{\lambda E\left[\left(1-\theta\left(e^{x}-1\right)\right)^{\gamma /(\gamma-1)}-1\right] T\right\} \\
& =\exp \left\{\frac{\gamma \theta^{2} \sigma^{2}}{2(\gamma-1)^{2}} T-\frac{\gamma r}{\gamma-1} T+\frac{\gamma}{\gamma-1} \theta \lambda E\left[\left(e^{x}-1\right)\right] T\right. \\
& \left.+\lambda E\left[\left(1-\theta\left(e^{x}-1\right)\right)^{\gamma /(\gamma-1)}-1\right] T\right\} .
\end{aligned}
$$


From (58), we have

$$
\begin{aligned}
\lambda^{1 /(\gamma-1)}=y_{0} \exp \{ & -\frac{\gamma \theta^{2} \sigma^{2}}{2(\gamma-1)^{2}} T+\frac{\gamma r}{\gamma-1} T-\frac{\gamma}{\gamma-1} \\
& \times \theta \lambda E\left[\left(e^{x}-1\right)\right] T \\
& \left.-\lambda E\left[\left(1-\theta\left(e^{x}-1\right)\right)^{\gamma /(\gamma-1)}-1\right] T\right\} .
\end{aligned}
$$

Then

$$
\begin{aligned}
& Y^{*}=\lambda^{1 /(\gamma-1)} H(T)^{1 /(\gamma-1)} \\
& =y_{0} \exp \left\{-\frac{\gamma \theta^{2} \sigma^{2}}{2(\gamma-1)^{2}} T+\frac{\gamma r}{\gamma-1} T\right. \\
& -\frac{\gamma}{\gamma-1} \theta \lambda E\left[\left(e^{x}-1\right)\right] T \\
& \left.-\lambda E\left[\left(1-\theta\left(e^{x}-1\right)\right)^{\gamma /(\gamma-1)}-1\right] T\right\} \\
& \cdot \exp \left\{-\frac{1}{\gamma-1} \theta \sigma W_{T}-\frac{1}{\gamma-1} r T-\frac{1}{2} \frac{1}{\gamma-1}\right. \\
& \times \theta^{2} \sigma^{2} T+\frac{1}{\gamma-1} \theta \lambda E\left[\left(e^{x}-1\right)\right] T \\
& \left.+\sum_{i=1}^{N(T)} \frac{1}{\gamma-1} \ln \left(1-\theta\left(e^{x_{i}}-1\right)\right)\right\} \\
& =y_{0} \exp \left\{-\frac{1}{\gamma-1} \theta \sigma W_{T}+r T\right. \\
& -\frac{(2 \gamma-1) \theta^{2} \sigma^{2}}{2(\gamma-1)^{2}} T-\theta \lambda E\left[\left(e^{x}-1\right)\right] T \\
& \left.-\lambda E\left[\left(1-\theta\left(e^{x}-1\right)\right)^{\gamma /(\gamma-1)}-1\right] T\right\} \\
& \cdot \exp \left\{\sum_{i=1}^{N(T)} \frac{1}{\gamma-1} \ln \left(1-\theta\left(e^{x_{i}}-1\right)\right)\right\} .
\end{aligned}
$$

In order to calculate the $\pi^{*}(t)$, from (57) and (58), we have

$$
Y^{*}=y_{0} \frac{H(T)^{1 /(\gamma-1)}}{E\left[H(T)^{\gamma /(\gamma-1)}\right]} .
$$

Thanks to the facts $Y^{*}=y^{*}(T)$ and $E_{t}\left[y^{*}(T) H(T)\right]=E_{t}\left[Y^{*}\right.$ $H(T)]$, then

$$
y^{*}(t) H(t)=E_{t}\left[Y^{*} H(T)\right]=y_{0} \frac{E_{t}\left[H(T)^{\gamma /(\gamma-1)}\right]}{E\left[H(T)^{\gamma /(\gamma-1)}\right]} .
$$

From (51), we have

$$
\begin{aligned}
& H(t)^{\gamma /(\gamma-1)} \\
& =\exp \left\{-\frac{\gamma}{\gamma-1} \theta \sigma W_{t}-\frac{\gamma}{\gamma-1} r t\right. \\
& -\frac{1}{2} \frac{\gamma}{\gamma-1} \theta^{2} \sigma^{2} t+\frac{\gamma}{\gamma-1} \theta \lambda E\left[\left(e^{x}-1\right)\right] t \\
& \left.+\sum_{i=1}^{N(t)} \frac{\gamma}{\gamma-1} \ln \left(1-\theta\left(e^{x_{i}}-1\right)\right)\right\} \\
& =\exp \left\{-\frac{\gamma}{\gamma-1} \theta \sigma W_{t}-\frac{\gamma^{2}}{2(\gamma-1)^{2}} \theta^{2} \sigma^{2} t\right. \\
& -\lambda E\left[\left(1-\theta\left(e^{x}-1\right)\right)^{\gamma /(\gamma-1)}-1\right] t \\
& \left.+\sum_{i=1}^{N(t)} \frac{\gamma}{\gamma-1} \ln \left(1-\theta\left(e^{x_{i}}-1\right)\right)\right\} \\
& \cdot \exp \left\{\frac{\gamma \theta^{2} \sigma^{2}}{2(\gamma-1)^{2}} t-\frac{\gamma r}{\gamma-1} t\right. \\
& +\frac{\gamma}{\gamma-1} \theta \lambda E\left[\left(e^{x}-1\right)\right] t \\
& \left.+\lambda E\left[\left(1-\theta\left(e^{x}-1\right)\right)^{\gamma /(\gamma-1)}-1\right] t\right\} .
\end{aligned}
$$

By denoting the martingale

$$
\begin{aligned}
z(t):=\exp \{ & -\frac{\gamma}{\gamma-1} \theta \sigma W_{t}-\frac{\gamma^{2}}{2(\gamma-1)^{2}} \theta^{2} \sigma^{2} t \\
& -\lambda E\left[\left(1-\theta\left(e^{x}-1\right)\right)^{\gamma /(\gamma-1)}-1\right] t \\
& \left.+\sum_{i=1}^{N(t)} \frac{\gamma}{\gamma-1} \ln \left(1-\theta\left(e^{x_{i}}-1\right)\right)\right\},
\end{aligned}
$$

and function

$$
\begin{aligned}
f(x):=\exp \left\{\frac{\gamma \theta^{2} \sigma^{2}}{2(\gamma-1)^{2}} t-\frac{\gamma r}{\gamma-1} t\right. \\
+\frac{\gamma}{\gamma-1} \theta \lambda E\left[\left(e^{x}-1\right)\right] t \\
\left.+\lambda E\left[\left(1-\theta\left(e^{x}-1\right)\right)^{\gamma /(\gamma-1)}-1\right] t\right\} .
\end{aligned}
$$

Then $H(t)^{\gamma /(\gamma-1)}$ can be written as

$$
H(t)^{\gamma /(\gamma-1)}=z(t) \cdot f(t) .
$$


From (63) and (67), we obtain

$$
\begin{aligned}
y^{*}(t) H(t) & =E_{t}\left[Y^{*} H(T)\right] \\
& =y_{0} \frac{E_{t}[z(T) f(T)]}{E[z(T) f(T)]}=y_{0} \frac{f(T) E_{t}[z(T)]}{f(T) E[z(T)]} \\
& =y_{0} \frac{E_{t}[z(T)]}{E[z(T)]}=y_{0} \frac{z(t)}{z(0)}=y_{0} z(t) .
\end{aligned}
$$

Hence,

$$
\begin{aligned}
& d\left(y^{*}(t) H(t)\right) \\
& =y_{0} d z(t) \\
& =y_{0} z(t)\left\{-\frac{\gamma}{\gamma-1} \theta \sigma d W_{t}\right. \\
& -\lambda E\left[\left(1-\theta\left(e^{x}-1\right)\right)^{\gamma /(\gamma-1)}-1\right] d t \\
& \left.+\left[\left(1-\theta\left(e^{x}-1\right)\right)^{\gamma /(\gamma-1)}-1\right] d N(t)\right\} \\
& =-y^{*}(t) H(t)\left\{\frac{\gamma}{\gamma-1} \theta \sigma d W_{t}\right. \\
& \quad+\lambda E\left[\left(1-\theta\left(e^{x}-1\right)\right)^{\gamma /(\gamma-1)}-1\right] d t \\
& \left.\quad-\left[\left(1-\theta\left(e^{x}-1\right)\right)^{\gamma /(\gamma-1)}-1\right] d N(t)\right\} .
\end{aligned}
$$

Comparing the diffusion of (69) and (51), we have

$$
\pi(t)=\frac{\theta}{1-\gamma} \text {. }
$$

\section{Conclusions}

With risk-minimization criterion, we employ the minimal martingale measure to solve the pricing problem in an incomplete market. Then we obtain the Radon-Nikodym derivative in the minimal martingale measure and a PIDE with respect to the European option. In a special case, we get the exact solution of European call option by Fourier transformation methods. Finally, we employ the pricing kernel to calculate the optimal portfolio selection by martingale methods.

\section{Acknowledgment}

This work is supported by the Fundamental Research Funds for the Central Universities (JBK130401).

\section{References}

[1] F. Black and M. Scholes, "The pricing of options and corporate liabilities," Journal of Political Economy, vol. 81, no. 3, pp. 637659, 1973.
[2] R. C. Merton, "Option pricing when underlying stock returns are discontinuous," Journal of Financial Economics, vol. 3, no. 1-2, pp. 125-144, 1976.

[3] G. Bakshi, C. Cao, and Z. Chen, "Empirical performance of alternative option pricing models," The Journal of Finance, vol. 52, no. 5, pp. 2003-2049, 1997.

[4] D. Bates, "The crash of '87: was it expected? The evidence from options markets," The Journal of Finance, vol. 46, no. 3, pp. 10091044, 1991.

[5] R. Cont and P. Tankov, Financial Modelling with Jump Processes, Financial Mathematics, Chapman \& Hall/CRC, Boca Raton, Fla, USA, 2004.

[6] S. G. Kou, "A jump-diffusion model for option pricing," Management Science, vol. 48, no. 8, pp. 1086-1101, 2002.

[7] K. I. Amin, "Jump diffusion option valuation in discrete time," The Journal of Finance, vol. 48, no. 5, pp. 1833-1863, 1993.

[8] D. Duffie, J. Pan, and K. Singleton, "Transform analysis and asset pricing for affine jump-diffusions," Econometrica, vol. 68, no. 6, pp. 1343-1376, 2000.

[9] J. E. Zhang, H. Zhao, and E. C. Chang, "Equilibrium asset and option pricing under jump diffusion," Mathematical Finance, vol. 22, no. 3, pp. 538-568, 2012.

[10] H. Föllmer and P. Leukert, "Efficient hedging: cost versus shortfall risk," Finance and Stochastics, vol. 4, no. 2, pp. 117-146, 2000 .

[11] J. Kallsen, "A utility maximization approach to hedging in incomplete markets," Mathematical Methods of Operations Research, vol. 50, no. 2, pp. 321-338, 1999.

[12] J. Cvitanić, W. Schachermayer, and H. Wang, "Utility maximization in incomplete markets with random endowment," Finance and Stochastics, vol. 5, no. 2, pp. 259-272, 2001.

[13] T. R. Bielecki and M. Jeanblanc, Indifference Pricing: Theory and Applications, Princeton Series in Financial Engineering, Edited by R. Carmona, Princeton University Press, Princeton, NJ, USA, 2009.

[14] M. Schweizer, “Option hedging for semimartingales," Stochastic Processes and Their Applications, vol. 37, no. 2, pp. 339-363, 1991.

[15] H. Föllmer and M. Schweizer, "Hedging of contingent claims under incomplete information," in Applied Stochastic Analysis, M. H. A. Davis and R. J. Elliott, Eds., vol. 5 of Stochastics Monographs, pp. 389-414, Gordon and Breach, London, UK, 1991.

[16] X. Ruan, W. Zhu, S. Li, and J. Huang, "Option pricing under risk-minimization criterion in an incomplete market with the finite difference method," Mathematical Problems in Engineering, vol. 2013, Article ID 165727, 9 pages, 2013.

[17] J. Pan, “The jump-risk premia implicit in options: evidence from an integrated time-series study," Journal of Financial Economics, vol. 63, no. 1, pp. 3-50, 2002.

[18] J. Liu and J. Pan, "Dynamic derivative strategies," Journal of Financial Economics, vol. 69, no. 3, pp. 401-430, 2003.

[19] J. Liu, J. Pan, and T. Wang, "An equilibrium model of rareevent premia and its implication for option smirks," Review of Financial Studies, vol. 18, no. 1, pp. 131-164, 2005.

[20] N. C. Framstad, B. Øksendal, and A. Sulem, "Sufficient stochastic maximum principle for the optimal control of jump diffusions and applications to finance," Journal of Optimization Theory and Applications, vol. 121, no. 1, pp. 77-98, 2004.

[21] G. Wang, Z. Wu, and J. Xiong, "Maximum principles for forward-backward stochastic control systems with correlated 
state and observation noises," SIAM Journal on Control and Optimization, vol. 51, no. 1, pp. 491-524, 2013.

[22] G. Wang and Z. Yu, "A partial information non-zero sum differential game of backward stochastic differential equations with applications," Automatica, vol. 48, no. 2, pp. 342-352, 2012.

[23] G. Wang and Z. Wu, "Mean-variance hedging and forwardbackward stochastic differential filtering equations," Abstract and Applied Analysis, vol. 2011, Article ID 310910, 20 pages, 2011.

[24] J. M. Harrison and D. M. Kreps, "Martingales and arbitrage in multiperiod securities markets," Journal of Economic Theory, vol. 20, no. 3, pp. 381-408, 1979.

[25] J. M. Harrison and S. R. Pliska, "A stochastic calculus model of continuous trading: complete markets," Stochastic Processes and Their Applications, vol. 15, no. 3, pp. 313-316, 1983.

[26] I. Karatzas, J. P. Lehoczky, S. E. Shreve, and G.-L. Xu, "Martingale and duality methods for utility maximization in an incomplete market," SIAM Journal on Control and Optimization, vol. 29, no. 3, pp. 702-730, 1991.

[27] H. He and N. D. Pearson, "Consumption and portfolio policies with incomplete markets and short-sale constraints: the infinite-dimensional case," Journal of Economic Theory, vol. 54, no. 2, pp. 259-304, 1991.

[28] G. Bakshi, N. Kapadia, and D. Madan, "Stock return characteristics, skew laws, and the differential pricing of individual equity options," Review of Financial Studies, vol. 16, no. 1, pp. 101-143, 2003.

[29] P. Carr and L. Wu, "Variance risk premiums," Review of Financial Studies, vol. 22, no. 3, pp. 1311-1341, 2009.

[30] A. Neuberger, "Realized skewness," Review of Financial Studies, vol. 25, no. 11, pp. 3423-3455, 2012. 


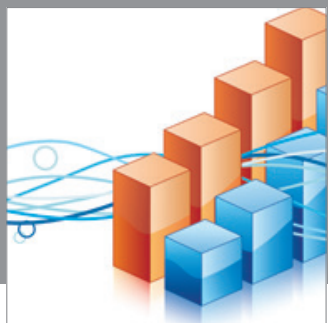

Advances in

Operations Research

mansans

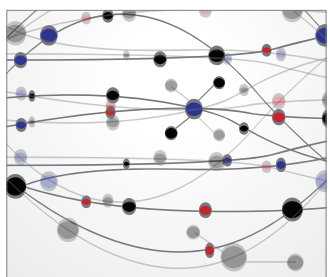

The Scientific World Journal
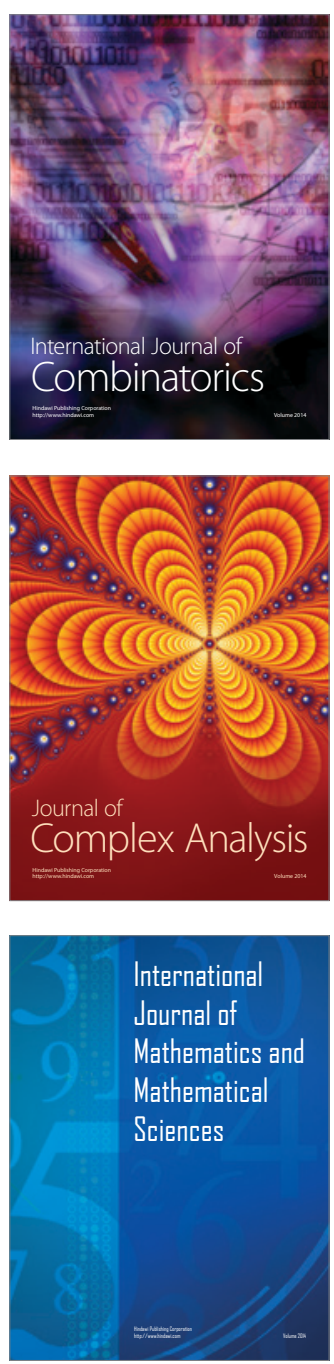
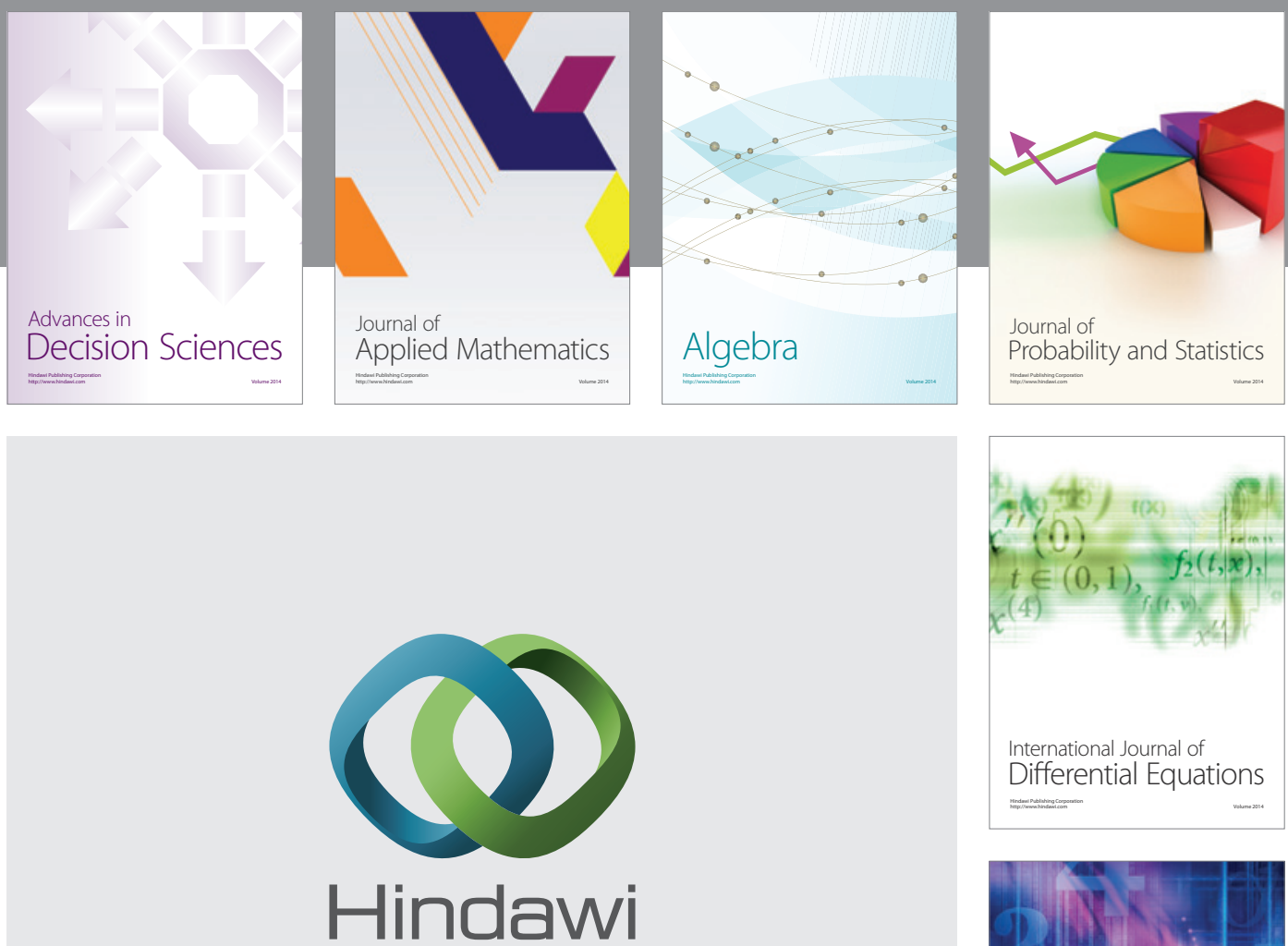

Submit your manuscripts at http://www.hindawi.com
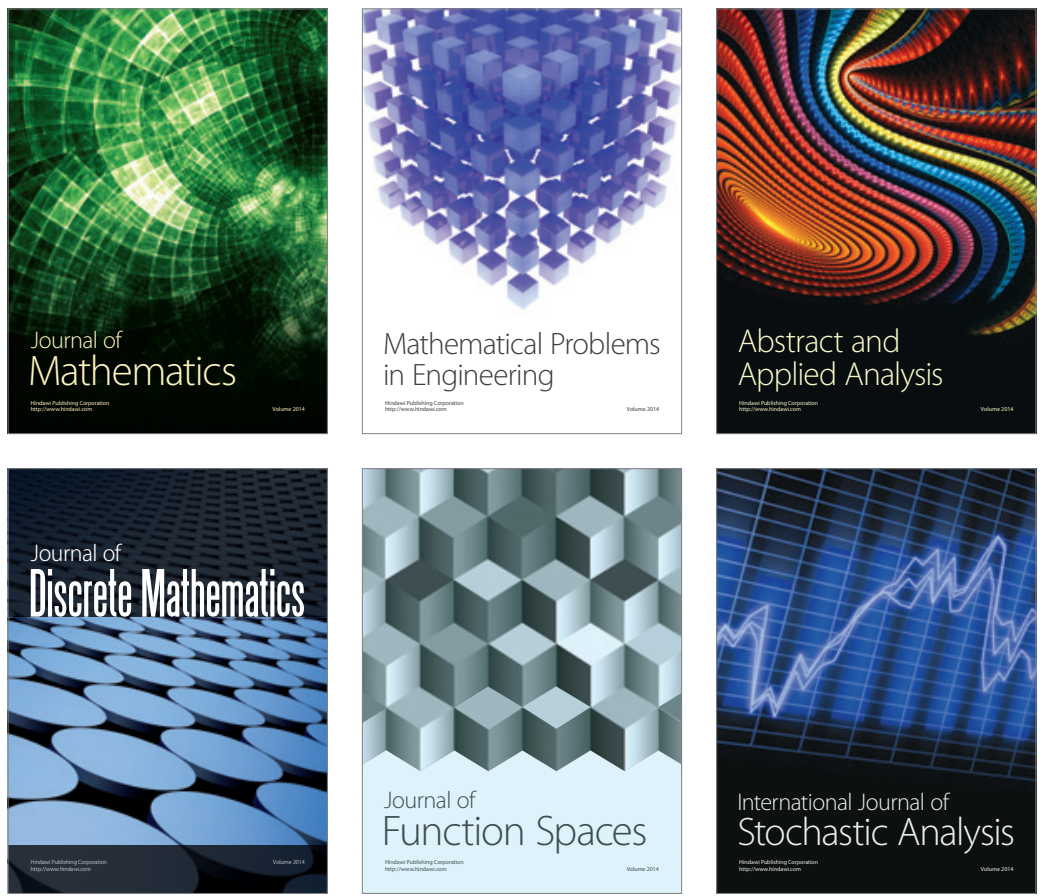

Journal of

Function Spaces

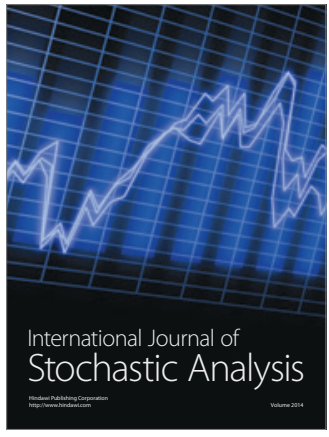

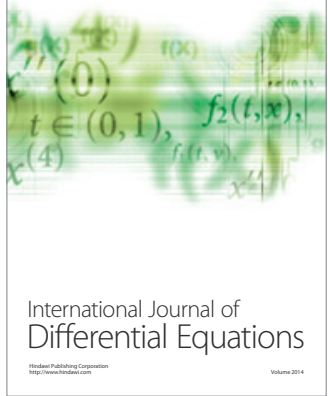
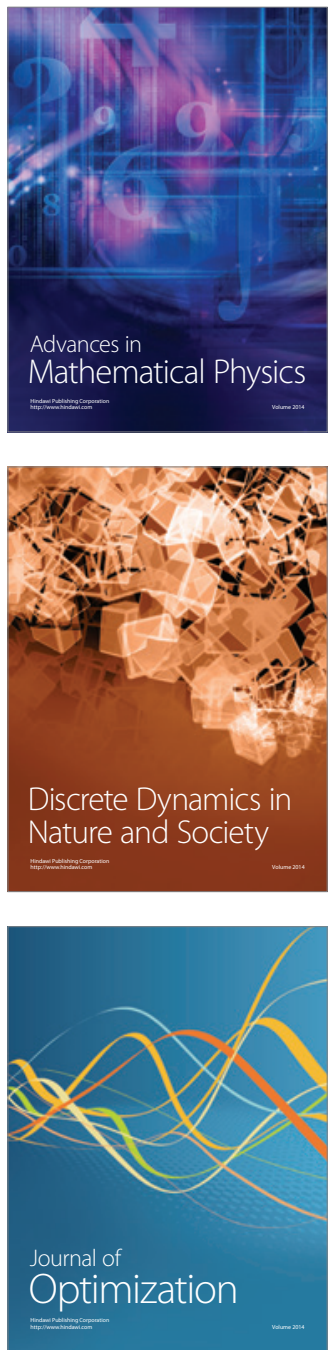